\title{
Night Vision Technology: An Overview
}

\author{
Mohd Junedul Haque \\ Department of Computer Science \\ College of Computers and Information Technology \\ Taif University \\ Saudi Arabia
}

\author{
Mohd Muntjir \\ Department of Information Technology \\ College of Computers and Information Technology \\ Taif University \\ Saudi Arabia
}

\begin{abstract}
Image Processing refers Capturing and manipulating images to enhance or extract information. Image processing is a form of signal processing for which the input is an image, such as a photograph or frame. The output of image processing may be either an image or, a set of characteristics or parameters related to the image. This paper is about Night vision Technology, by definition, literally allows one to see in the dark, originally developed for military use. Night vision can work in two very different ways, depending on the technology used. Image enhancement-This works by using the lower portion of the infrared light spectrum. Thermal imaging - This technology operates by using the upper portion of the infrared light spectrum.
\end{abstract}

\section{Keywords}

Night vision Technology, Image enhancement, Image intensifier tube, Thermal imaging (Un-cooled, Cryogenically cooled), near infrared, Mid-infrared, Thermal infrared.

\section{INTRODUCTION}

Night Vision technology:

Night vision technology was developed by the US defense department mainly for defense purposes, but with the development of technology night vision devices are being used in day to day lives. Night Vision can work in two different ways depending on the technology used.

1. Image enhancement- This works by collecting the tiny amounts of light including the lower portion of the infrared light spectrum, those are present but may be imperceptible tour eyes, and amplifying it to the point that we can easily observe the image.

2. Thermal imaging- This technology operates by capturing the upper portion of the infrared light spectrum, which is emitted as heat by objects instead of simply reflected as light. Hotter objects, such as warm bodies, emit more of this light than cooler objects like trees or buildings.

The Basics:

In order to understand night vision, it is important to understand something about light. The amount of energy in alight wave is related to its wavelength. Shorter wavelengths have higher energy. Of visible light, violet has the most energy, and red has the least. Just Next to the visible light spectrums the infrared spectrum. Infrared light can be split into three categories:

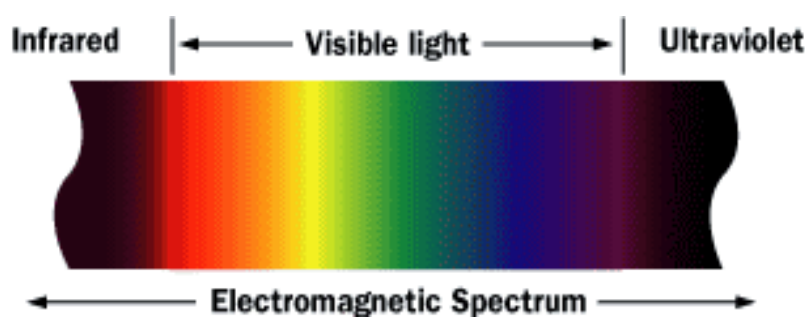

Fig 1.1

Near-infrared (near-IR) - Closest to visible light, near-IR has wavelengths that range from 0.7 to 1.3 microns, or 700billionths to 1,300 billionths of a meter.

Mid-infrared (mid-IR) - Mid-IR has wavelengths ranging from1.3 to 3 microns. Both near-IR and mid-IR are used by a variety of electronic devices, including remote controls.

Thermal-infrared (thermal-IR) - Occupying the largest part of the infrared spectrum, thermal-IR has wavelengths ranging from 3 microns to over 30 microns.

The key difference between thermal-IR and the other two is that thermal-R is emitted by an object instead of reflected off it. Infrared light is emitted by an object because of what is happening at the atomic level.

\section{Atoms:}

Atoms are constantly in motion. They continuously vibrate, move and rotate. Even the atoms that make up the chairs that we sit in are moving around. Solids are actually in motion! Atoms can be in different states of excitation. In other words, they can have different energies. If we apply a lot of energy to an atom, it can leave what is called the ground-state energy level and move to an excited level. The level of excitation depends on the amount of energy applied to the atom via heat, light or electricity. An atom consists of a nucleus (containing the protons and neutrons) and an electron cloud. Think of the electrons in this cloud as circling the nucleus in many different orbits. Although more modern views of the atom do not depict discrete orbits for the electrons, it can be useful to think of these orbits as the different energy levels of the atom. In other words, if we apply some heat to an atom, we might expect that some of the electrons in the lower energy orbitals would transition to higher energy orbitals, moving farther from the nucleus. 


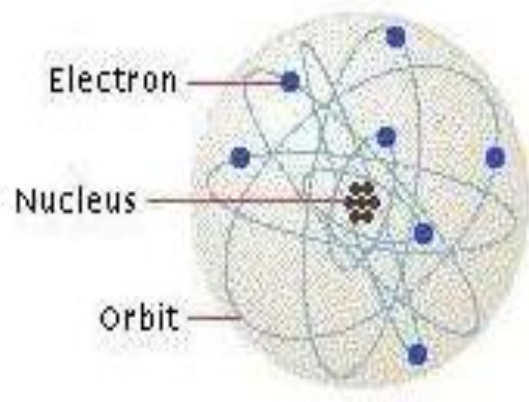

Fig 1.2

Once an electron moves to a higher-energy orbit, it eventually wants to return to the ground state. When it does, it releases its energy as a photon a particle of light. You see atoms releasing energy as photons all the time. For example, when the heating element in a toaster turns bright red, the red color is caused by atoms excited by heat, releasing red photons. An excited electron has more energy than a relaxed electron, and just as the electron absorbed some amount of energy to reach this excited level, it can release this energy to return to the ground state. This emitted energy is in the form of photons (light energy). The photon emitted has a very specific wavelength (color) that depends on the state of the electron's energy when the photon is released.

\section{A. Thermal Imaging and Image Enhancement:} Here's how thermal imaging works:

A special lens focuses the infrared light emitted by all of the objects in view.

The focused light is scanned by phased array of infrared-detector elements. The detector elements create a very detailed temperature pattern called a thermo gram. It only takes about one-thirtieth of a second for the detector array to obtain the temperature information to make the thermo gram. This information is obtained from several thousand points in the field of view of the detector array.

The thermo gram created by the detector elements is translated into electric impulses.

The impulses are sent to a signal-processing unit, a circuit board with a dedicated chip that translates the information from the elements into data for the display.

The signal-processing unit sends the information to the display, where it appears as various colors depending on the intensity of the infrared emission. The combination of all the impulses from all of the elements creates the image.

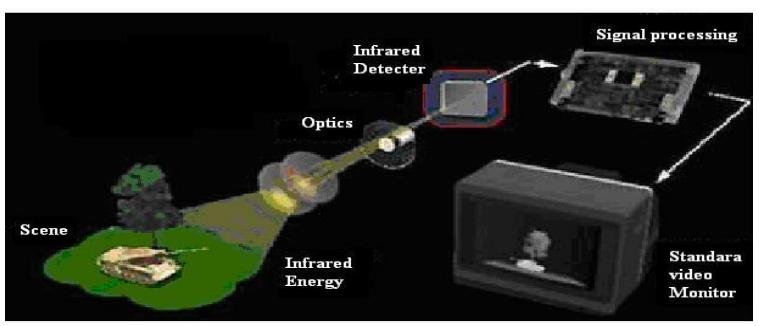

Fig 1.3

There are two common types of thermal-imaging devices:
Un-cooled - This is the most common type of thermal-imaging device. The infrared-detector elements are contained in a unit that operates at room temperature. This type of system is completely quiet, activates immediately and has the battery built right in.

Cryogenically cooled - More expensive and more susceptible to damage from rugged use, these systems have the elements sealed inside a container that cools them to below $32 \mathrm{~F}$ (zeroC). The advantage of such a system is the incredible resolution nd sensitivity that results from cooling the elements. Cryogenicallycooled systems can "see" a difference as small as $0.2 \mathrm{~F}(0.1 \mathrm{C})$ from more than $1,000 \mathrm{ft}(300 \mathrm{~m})$ away, which is enough to tell if a person is holding a gun at that distance.

\section{B. Image Enhancement:}

Image-enhancement technology is what most people think of when you talk about night vision. In fact, image-enhancement systems are normally called night-vision devices (NVDs).NVDs rely on a special tube, called an image-intensifier tube, to collect and amplify infrared and visible light.

Here's how image enhancement works

A conventional lens, called the objective lens, captures ambient light and some near-infrared light.

The gathered light is sent to the image-intensifier tube. In most NVDs, the power supply for the image testifier tube receives power from two N-Cell or two "A" batteries. The tube outputs a high voltage, about 5,000 volts, to the image-tube components.

The image-intensifier tube has a photocathode, which his used to convert the photons of light energy into electrons.

As the electrons pass through the tube, similar electrons are released from atoms in the tube, multiplying the original number of electrons by a factor of thousands through the use of a micro channel plate (MCP) in the tube. An MCP is a tiny glass dis chat has millions of microscopic holes (micro channels) in it, made using fiber-optic technology. The MCP is contained in a vacuum and has metal electrodes on either side of the disc. Each channel is about 45 times longer than it is wide, and it works as an electron multiplier. When the electrons from the photo cathode hit the first electrode of the MCP, they are accelerated into the glass micro channels by the 5,000-V bursts being sent between the electrode pair. As electrons pass through the micro channels, they cause thousands of other electrons to be released in each channel using a process called cascaded secondary emission. Basically, the original electrons collide with the side of the channel, exciting atoms and causing other electrons to be released. These new electrons also collide with other atoms, creating a chain reaction that results in thousands of electrons leaving the channel where only a few entered. An interesting fact is that the micro channels in the MCP are created at a slight angle (about a5-degree to 8-degree bias) to encourage electron collisions and reduce both ion and direct-light feedback from the phosphors on the output side.

At the end of the image- intensifier tube, the electrons hit a screen coated with phosphors. These electrons maintain their position in relation to the channel they passed through, which provides a perfect image since the electrons stay in the same alignment as the original photons. The energy of the electrons causes the phosphors to reach an excited state and release photons. These phosphors create the green image on the screen that has come to characterize night vision. 


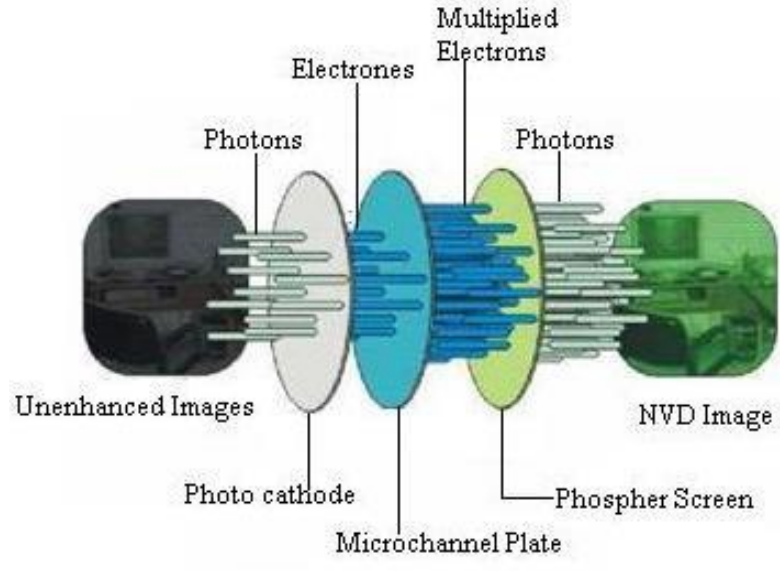

Fig 1.4

The green phosphor image is viewed through another lens, called the ocular lens, which allows you to magnify and focuses the image. The NVD may be connected to an electronic display, such as a monitor, or the image may be viewed directly through the ocular lens.

\section{Characteristics of Night Vision:}

Using intensified night vision is different from using regular binoculars and/or your own eyes. Below are some of the aspects of night vision that you should be aware of when you are using an image intensified night vision system.

Textures, Light and Dark:

Objects that appear light during the day but have a dull surface may appear darker, through the night vision unit, than objects that are dark during the day but have a highly reflective surface. For example, a shiny dark colored jacket may appear brighter than a light colored jacket with a dull surface.

Depth Perception:

Night vision does not present normal depth perception.

Fog and Rain:

Night vision is very responsive to reflective ambient light; therefore, the light reflecting off of fog or heavy rain causes much more light to go toward the night vision unit and may degrade its performance.

Honeycomb:

This is a faint hexagonal pattern which is the result of the manufacturing process.

\section{Black Spots:}

A few black spots throughout the image area are also inherent characteristics of all night vision technology. These spots will remain constant and should not increase in size or number. See example below of an image with black spots. [Fig 1.1]

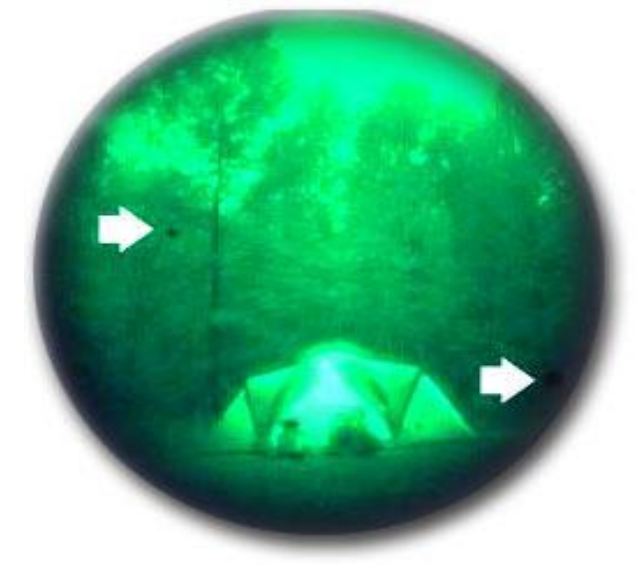

Fig 1.5

Blooming:

Loss of the entire night vision image, parts of it, or small parts of it, due to intensifier tube overloading by a bright light source. Also, known as a "halo" effect, when the viewer sees a "halo" effect around visible light sources. When such a bright light source comes into the night vision device's view, the entire night vision scene, or parts of it, becomes much brighter, "whiting out" objects within the field of view. Blooming is common in Generation 0 and 1 devices. The lights in the image to the right would be considered to be "blooming".

Bright-Source Protection (BSP):

High-Light Cut-Off, an electronic function that reduces the voltage to the photocathode when the night vision device is exposed to bright light sources such as room lights or carlights.BSP protects the image tube from damage and enhances its life; however, it also has the effect of lowering resolution when functioning.

\section{Chicken Wire:}

An irregular pattern of dark thin lines in the field of view either throughout the image area or in parts of the image area. Under the worst-case condition, these lines will form hexagonal or square wave-shape lines.

\section{Daylight Lens Cover:}

Usually made of soft plastic or rubber with a pin hole that allows a small amount of light to enter the objective lens of a night vision device. This should be used for training purposes only, and is not recommended for an extended period of time.

Daylight Training Filter:

A glass filter assembly designed to fit over the objective lens of a night vision device. The filter reduces light input to a safe (night-time) level, allowing safe extended daytime use of the night vision device.

Equivalent Background Illumination (EBI):

This is the amount of light you see through a night vision device when an image tube is turned on but no light is on the photo cathode. EBI is affected by temperature; the warmer the night vision device, the brighter the background illumination.EBI is measured in lumens per square centimeter $(\mathrm{lm} / \mathrm{cm} 2)$. The lower the value the better. The EBI level determines the lowest light 
level at which an image can be detected. Below this light level, objects will be masked by the EBI.

\section{Emission Point:}

A steady or fluctuating pin point of bright light in the image area that does not go away when all light is blocked from the objective lens. The position of an emission point within the field of view will not move. If an emission point disappears or is only faintly visible when viewing under brighter night time conditions, it is not indicative of a problem. If the emission point remains bright under all lighting conditions, the system needs to be repaired. Do not confuse an emission point with a point of light source in the scene being viewed.

\section{Equipment and Applications:}

Night-vision equipment can be split into three broad categories: Scopes - Normally handheld or mounted on a weapon, scopes are monocular (one eye-piece). Since scopes are handheld, not worn like goggles, they are good for when you want to get a better look at a specific object and then return to normal viewing conditions.

Goggles - While goggles can be handheld, they are most often worn on the head. Goggles are binocular (two eye-pieces) and may have a single lens or stereo lens, depending on the model. Goggles are excellent for constant viewing, such as moving around in a dark building.

Cameras - Cameras with night-vision technology can send the image to a monitor for display or to a VCR for recording. Then night-vision capability is desired in a permanent location, such as on a building or as part of the equipment in a helicopter, cameras are used. Many of the newer camcorders have night vision built right in.

\section{E. Applications:}

Common applications for night vision include:

- Military

- Law enforcement

- Hunting

- Wildlife observation

- Surveillance

- Security

- Navigation

- Hidden-object detection

The original purpose of night vision was to locate enemy targets at night. It is still used extensively by the military for that purpose, as well as for navigation, surveillance and targeting. Police and security often use both thermal- imaging and imageenhancement technology, particularly for surveillance. Hunters and nature enthusiasts use NVDs to maneuver through the woods at night. Detectives and private investigators use night vision to watch people they are assigned to track. Many businesses have permanently-mounted cameras equipped with night vision to monitor the surroundings.

A really amazing ability of thermal imaging is that it reveals whether an area has been disturbed -- it can show that the ground has been dug up to bury something, even if there is no obvious sign to the naked eye. Law enforcement as used this to discover items that have been hidden by criminals, including money, drugs and bodies. Also, recent changes to areas such as walls can be seen using thermal imaging, which has provided important clues in several cases.

\section{Gallium Arsenide (GaAs):}

The semiconductor material used in manufacturing the Gen 3 photocathode.GaAs photo cathodes have a very high photo sensitivity in the spectral region of about 450 to 950 nanometers (visible and near-infrared region). High light shut off an image intensifier protection feature incorporating a sensor, microprocessor and circuit breaker. This feature will turn the system off during periods of extreme bright light conditions.

Interpupillary Distance:

The distance between the user's pupils (eyeball centers). The 95th percentile of US military personnel falls within the 55 to $72 \mathrm{~mm}$ range of IPD.

\section{IR Illuminator:}

Many nights vision devices incorporate a built-in infrared (IR) diode that emits invisible light or the illuminator can be mounted on to it as a separate component. IR light cannot be seen by the unaided eye; therefore, a night vision device is necessary to see this light. IR Illuminators provide supplemental infrared illumination of an appropriate wavelength, typically in a range of wavelengths (e.g. $730 \mathrm{~nm}, 830 \mathrm{~nm}, 920 \mathrm{~nm}$ ), and eliminate the variability of available ambient light, but also allow the observer to illuminate only specific areas of interest while eliminating shadows and enhancing image contrast.

IR Laser:

High-power devices providing long-range illumination capability. Ranges of several thousand meters are common. Most are not eye-safe and are restricted in use. Each IR laser should be marked with a warning label like the one shown here. Consult FDA CFR Title 21 for specific details and restrictions.

\section{I2 (Image Intensification):}

Collects and intensifies the available light in the visible and near-infrared spectrum. Offers a clear, distinguishable image under low-light conditions.

\section{RELATED WORKS}

A night vision device (NVD) is an optical instrument that allows images to be produced in levels of light approaching total darkness. They are most often used by the military and law enforcement agencies, but are available to civilian users. The term usually refers to a complete unit, including an image intensifier tube, a protective and generally water-resistant housing, and some type of mounting system. Many NVDs also include sacrificial lenses, IR illuminators, and telescopic lenses. Night vision devices were first used in World War II, and came into wide use during the Vietnam War.[1][2]The technology has evolved greatly since their introduction, leading to several "generations" of night vision equipment with performance increasing and price decreasing.

\section{Generations}

NVDs have been around for more than 40 years. They are categorized by generation. Each substantial change in NVD technology establishes a new generation.

Generation 0 - The original night-vision system created by the United States Army and used in World War II and the Korean War, these NVDs use active infrared. This means that a projection unit, called an IR Illuminator, is attached to the NVD. The unit projects a beam of near-infrared light, similar to the beam of a normal flashlight. Invisible to the naked eye, this beam reflects off objects and bounces back to the lens of the NVD. These systems use an anode in conjunction with the cathode to accelerate the electrons. The problem with that approach is that the acceleration of the electrons distorts the image and greatly decreases the life of the tube. Another major problem with this technology in its original military use was that it was quickly duplicated by hostile nations, which allowed enemy soldiers to use their own NVDs to see the infrared beam projected by the device. 
Generation 1 - The next generation of NVDs moved away from active infrared, using passive infrared instead. Once dubbed Starlight by the U.S. Army, these NVDs use ambient light provided by the moon and stars to augment the normal amounts of reflected infrared in the environment. This means that they did not require a source of projected infrared light. This also means that they do not work very well on cloudy or moonless nights. Generation-1 NVDs use the same image-intensifier tube technology as Generation 0, with both cathode and anode, so image distortion and short tube life are still a problem.

Generation 2 - Major improvements in image-intensifier tubes resulted in Generation-2 NVDs. They offer improved resolution and performance over Generation-1 devices, and are considerably more reliable. The biggest gain in Generation 2 is the ability to see in extremely low light conditions, such as a moonless night. This increased sensitivity is due to the addition of the micro channel plate to the image-intensifier tube. Since the MCP actually increases the number of electrons instead of just accelerating the original ones, the images are significantly less distorted and brighter than earlier-generation NVDs.

Generation 3 - Generation 3 is currently used by the U.S. military. While there are no substantial changes in the underlying technology from Generation 2, these NVDs have even better resolution and sensitivity. This is because the photo cathode is made using gallium arsenide, which is very efficient at converting photons to electrons. Additionally, the MCP is coated with an iron barrier, which dramatically increases the life of the tube.

Generation 4 - What is generally known as Generation 4 or "filmless and gated" technology shows significant overall improvement in both low- and high-level light environments.

The removal of the ion barrier from the MCP that was added in Generation 3 technology reduces the background noise and thereby enhances the signal to noise ratio. Removing the ion film actually allows more electrons to reach the amplification stage so that the images are significantly less distorted and brighter.

The addition of an automatic gated power supply system allows the photocathode voltage to switch on and off rapidly, thereby enabling the NVD to respond to a fluctuation in lighting conditions in an instant. This capability is a critical advance in NVD systems, in that it allows the NVD user to quickly move from high-light to low-light (or from low-light to high-light) environments without any halting effects. For example, consider the ubiquitous movie scene where an agent using night vision goggles is "sightless" when someone turns on a light nearby. With the new, gated power feature, the change in lighting wouldn't have the same impact; the improved NVD would respond immediately to the lighting change.

Many of the so-called "bargain" night-vision scopes use Generation-0 or Generation-1 technology, and may be disappointing if you expect the sensitivity of the devices used by professionals. Generation-2, Generation-3 and Generation 4 NVDs are typically expensive to purchase, but they will last if properly cared for. Also, any NVD can benefit from the use of an IR Illuminator in very dark areas where there is almost no ambient light to collect.

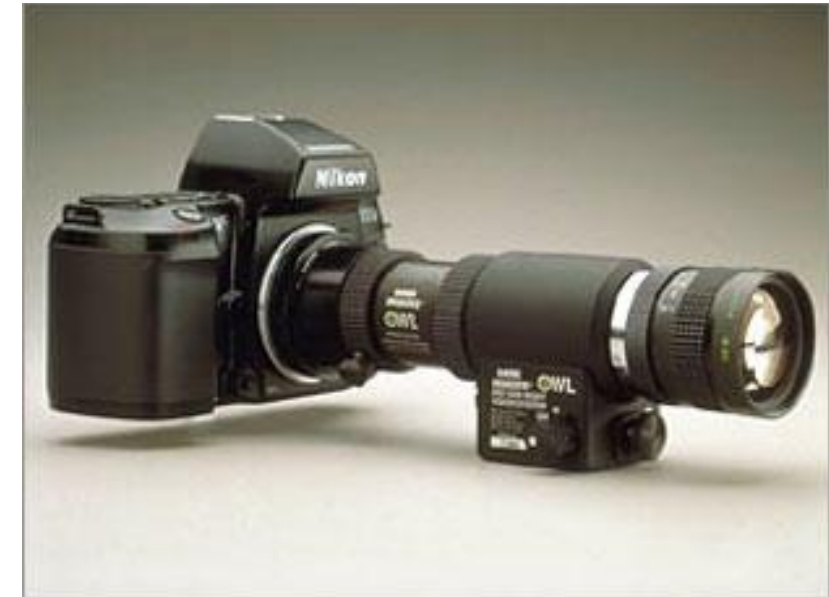

Fig 2.1

Photo courtesy of B.E. Meyers Company

NVDs come in a variety of styles, including ones that can be mounted to cameras.

A cool thing to note is that every single image-intensifier tube is put through rigorous tests to see if it meets the requirements set forth by the military. Tubes that do are classified as MILSPEC. Tubes that fail to meet military requirements in even a single category are classified as COMSPEC.

\section{CONCLUSION}

In this paper we have described various night vision technologies which are available and also its working in order to avoid various low light problem, this paper shows that how efficiently a soldiers can work efficiently during night also wildlife observer can work during dark and also shown how surveillance can be kept in low light condition.

The Night vision device (NVD) is undoubtedly one of the most enduring features of the automobile industry. It has lasted from its initial introduction in 2000 to the present day and in some places, still hardly different from its Victorian origins. There have been many improvements over the years without any skill required.

\section{REFERENCES}

[1] www.Explainthatstuff.com.

[2] Hussain, Z. "Digital Image Processing". Practical Applications of Parallel Processing Techniques. Published by: Ellis Horwood Limited, 1991.

[3] Parker, J., R., "Algorithms for Image Processing and Computer Vision”, Wiley Computer Publishing, 1997.

[4] Parker, J., R., "Practical Computer Vision using C", Wiley Computer Publishing, 1994.

[5] Ritter, G., X., Wilson, J., N., "Handbook of Computer Vision Algorithms in Image Algebra", CRC Press, 1996.

[6] Russ, J. C., "The Image Processing Handbook". CRC Press, 1992.

[7] Sanz, J. L. "Advances in Machine Vision”, Spring-Verlag, 1989.

[8] http://www.bps.go.id

[9] www.amswer.com

[10] www.nightvision.com

[11] Belhumeur, P., hespanha, J., and Kriegman, D., "Eigen faces vs. Fisher faces 
[12] Recognition Using Class Specific Linear Projection", IEEE Trans. Pattern Analysis Machine Intelligence, vol. 19, no. 7, pp.711-720, July 1997.

[13] Brunelli, R., and Poggio, T., "Face Recognition: Features versus Templates", IEEE Trans. Pattern Anal. Machine Intell. vol. 15, pp.1042-1052,1993.

[14] Chan, Y., Lin, S.-H., and Kung, S.Y., "Video Indexing and Retrieval "
[15] Li, H., Roivainen, P., and Forchheimer, R., "3-D Motion Estimation in Model-Based Facial Image Coding", IEEE Trans. Pattern Analysis and Machine Intelligence, vol. 15, no. 6, pp.545-555, 1993.

[16] Pentland, A.P., Moghaddam, B., Starner, T., and Turk, M.A., "View based and Modular Eigen spaces for Face Recognition”, Proc. IEEE Lin, S.-H., Kung, S.Y., and Lin, L.-J., "Face Recognition/Detection by Probabilistic Decision-Based Neural Network". 